\title{
Impact of moral sensitivity on moral distress among psychiatric nurses
}

\section{Ohnishi, Kayoko}

2019-08

Ohnishi , K , Kitaoka , K , Nakahara , J , Välimäki , M , Kontio , R \& Anttila , M 2019 , ' Impact of moral sensitivity on moral distress among psychiatric nurses ' , Nursing Ethics , vol. 26 , no. 5 , pp. 1473-1483 . https://doi.org/10.1177/0969733017751264

http://hdl.handle.net/10138/320947

https://doi.org/10.1177/0969733017751264

publishedVersion

Downloaded from Helda, University of Helsinki institutional repository.

This is an electronic reprint of the original article.

This reprint may differ from the original in pagination and typographic detail.

Please cite the original version. 


\title{
Impact of moral sensitivity on moral distress among psychiatric nurses
}

\section{Kayoko Ohnishi}

Konan Women's University, Japan

\author{
Kazuyo Kitaoka \\ Kanazawa University, Japan
}

\section{Jun Nakahara}

Seigakuin University, Japan

\author{
Maritta Välimäki \\ University of Turku, Finland and Turku University Hospital, Finland
}

\section{Raija Kontio}

Hospital District of Helsinki and Uusimaa, Finland

\section{Minna Anttila}

University of Turku, Finland

\begin{abstract}
Background: Moral distress occurs when one knows the right thing to do, but institutional constraints make it nearly impossible to pursue the right course of action. Moral distress was found to cause negative feelings, burnout, and/or resignation. Not only external factors such as lack of staff but also internal ones affect moral distress. Moral sensitivity, which is thought of as an advantage of nurses, could effect moral distress, as nurses being unaware of existing ethical problems must feel little distress.

Objectives: To examine the impact of moral sensitivity on moral distress among psychiatric nurses, and affirm the hypothesis that nurses with higher moral sensitivity will suffer moral distress more than nurses with less moral sensitivity in two different samples.

Ethical consideration: The study obtained ethical approval from the Research Ethics Committee of the Faculty of Medicine at Mie University (\# IIII, 20.4.20I0), and by the Turku University Ethics Board (29.5.2012). Permissions to undertake the study was obtained from the in two hospital districts and in one city $(\S 48 / 4$. 10.20I2, $\S 63 / 4.9 .2012,5 \mathrm{I} / 201227.8 .2012)$. Informed consent was not formally obtained, because the questionnaire was anonymously reported by the participants who volunteered to answer. The participants responded voluntarily and anonymously.

Methods: An anonymous questionnaire containing the Revised Moral Sensitivity Questionnaire and the Moral Distress Scale for Psychiatric nurses was conducted to 997 nurses in 12 hospitals in Japan, and 974 nurses in 10 hospitals in Finland after obtaining of approval by research ethics committees. Data were analyzed using a multi-group structural equation model analysis.
\end{abstract}


Findings: A set of analyses imply that the association of moral sensitivity with moral distress is significant and similar between Japan and Finland, whereas the factor structures of moral sensitivity and moral distress may be partially different.

Discussion: The result of this study may indicate that nurses with high moral sensitivity can sense and identify moral problems, but not resolve them. Therefore, supporting nurses to solve ethical problems, not benumbing them, can be important for better nursing care and prevention of nurses' resignation.

Conclusion: Moral sensitivity and moral distress were positively correlated among psychiatric nurses in both Japan and Finland, although the participating nurses from the two countries were different in qualification, age, and cultural background. Nurses with high moral sensitivity suffer from moral distress.

\section{Keywords}

Model testing, moral distress, moral sensitivity, psychiatric nurse

\section{Introduction}

Moral distress, first described by Jameton ${ }^{1}$ in 1984 , occurs when one knows the right thing to do, but institutional constraints make it nearly impossible to pursue the right course of action. Moral distress has increasingly attracted attention mainly on its adverse effects since then. It can produce a variety of negative feelings such as anger, frustration, or guilt, and long-term moral distress may be detrimental to nurses' personal or professional well-being. ${ }^{2}$ It is also related to job stress, job dissatisfaction, ${ }^{3}$ burnout, ${ }^{4}$ and turnover. ${ }^{5}$

Poor working condition, such as a lack of adequate staff, may lead to inappropriate care and result in nurses experiencing moral distress. ${ }^{6}$ External factors such as working environment are not, however, the only determinants of whether or not moral distress occurs because psychiatric nurses in two different countries with varying nurse-patient ratios and other conditions experienced moral distress with similar intensity. ${ }^{7}$

Moral distress among nurses can be presumed to be affected by internal factors, including religious beliefs, and family values. ${ }^{8}$ The number of studies that focus on the relationship between moral sensitivity and moral distress has increased recently. ${ }^{9}$ For example, Nejadsarvari et al. ${ }^{10}$ revealed that there is a significant negative relationship between moral sensitivity and the frequency of moral distress, and significant positive relationship between moral sensitivity and the intensity of moral distress experienced by physicians in Tehran. Physicians with higher moral sensitivity feel moral distress more intensely, while physicians that experience moral distress frequently might adjust to the distressing situations, and consequently become less sensitive. Escolar-Chua ${ }^{11}$ administered a survey to undergraduate nursing students in the Philippines and showed a significant positive association between moral distress intensity and moral sensitivity. Borhani et al., ${ }^{12}$ however, found no meaningful statistical relationship between moral sensitivity and moral distress among nurses in critical care units.

The relationship between moral sensitivity and moral distress among psychiatric nurses has not been explored empirically. The topic is important in this group because specific ethical issues exist in psychiatric settings that contribute to moral distress. Examples of these include involuntary commitment, inappropriate hospitalization, and neglectful or abusive treatment. ${ }^{13}$ Psychiatric patients are vulnerable; they may be disliked, feared, persecuted, or stigmatized because of their mental illness. ${ }^{14}$ Their competence for decisionmaking has not been regarded reliable, ${ }^{15}$ and patients with mental health problems have historically experienced deprivation of many rights enjoyed by other citizens. ${ }^{14}$

\section{Purposes and hypothesis}

This study aimed to examine the impact of moral sensitivity on moral distress among psychiatric nurses. The hypothesis is that moral sensitivity is positively associated with modal distress among psychiatric 


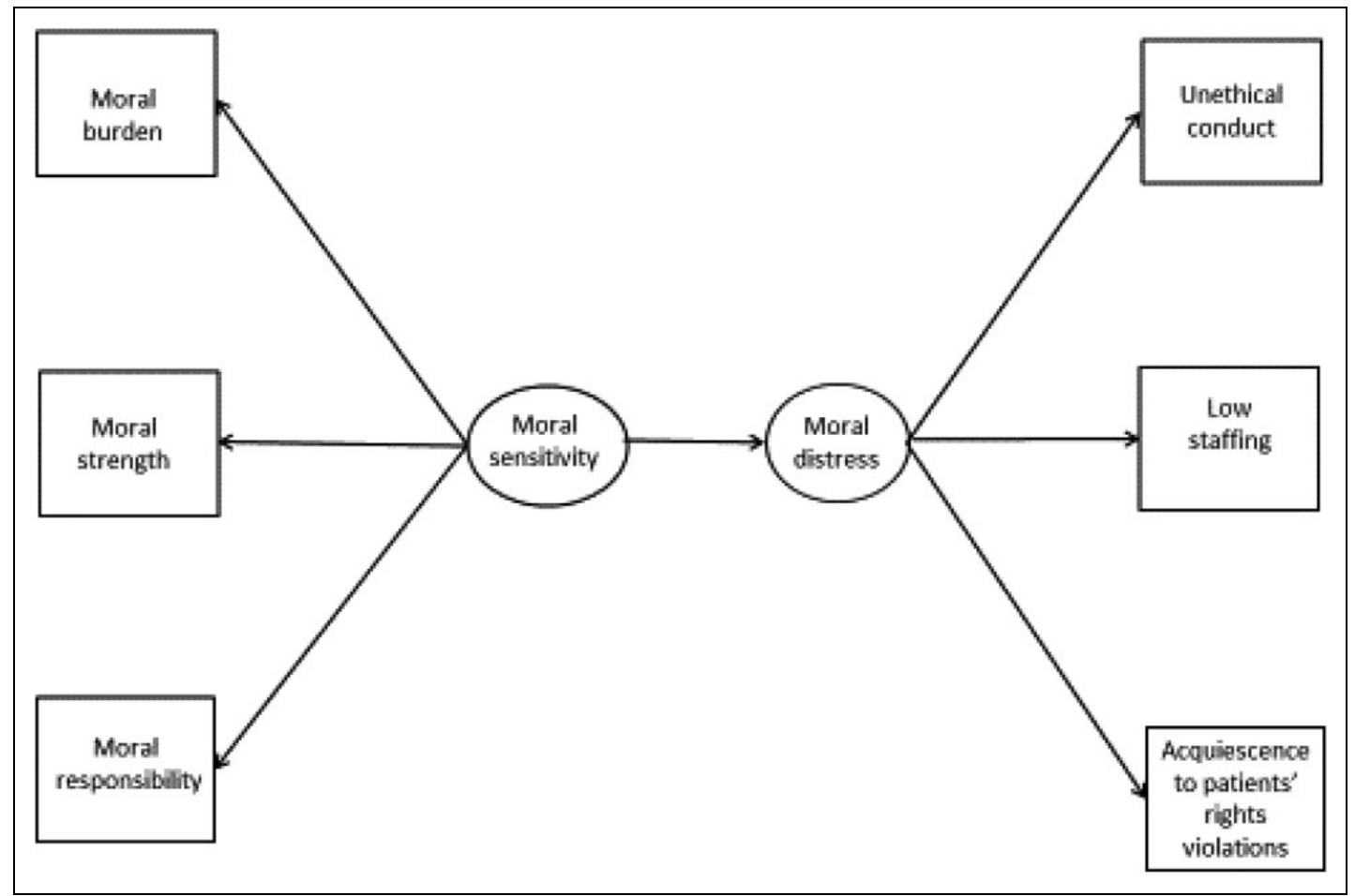

Figure I. Hypothesis of the relationship between moral sensitivity and moral distress.

nurses; that is nurses with higher moral sensitivity will suffer greater moral distress than those with less moral sensitivity (Figure 1).

Moral distress experienced by psychiatric nurses has been suggested to vary with cultures, due to the empirical diversity of practices and perspectives in beliefs and values in addition to languages and customs. ${ }^{16}$ If the relationship between moral sensitivity and moral distress is similar between countries with different cultures, then the relationship between moral sensitivity and moral distress could be more general than originally thought.

\section{Methods}

\section{Design}

The study used cross-sectional design with a self-administered questionnaire, a method useful for investigating associations between risk factors and outcome of interest. ${ }^{17}$

\section{Setting}

The study was conducted at the same time in two countries: Japan and Finland. These two countries were selected because they represent different cultures including religions, beliefs and values, and working conditions.

The Japanese study included 12 psychiatric hospitals in six prefectures, located in urban and rural areas. Among the hospitals, four were public (national or municipal) and eight were private. One public hospital 
was a general hospital that had only one psychiatric ward, while others were psychiatric hospitals, each with two to nine wards. These hospitals had a total of 48 wards, which were either acute, chronic, or rehabilitation wards, and more than half of them were locked all day. There are approximately 50 beds (patients) in each ward. Almost all of the inpatients were adults and older persons.

In Finland, 10 public hospitals with psychiatric beds in two hospital districts and in one city were recruited to participate in the study. These hospitals had a total of 36 wards participating in the study, which were open $24 \mathrm{~h}$ a day and were either acute or rehabilitation wards, caring for adult, adolescents, and older persons.

\section{Target population and inclusion and exclusion criteria}

The target population of the study was nurses who worked in psychiatric wards. In Japan, the ratio of nurses to inpatients in psychiatric wards is lower than the ratio in general wards. ${ }^{18}$ Among the nurses working in psychiatric hospitals, approximately two-thirds are registered nurses (RNs), including very few certified nurse specialists (CNSs), and the others are assistant nurses. ${ }^{18}$ The rate of psychiatric nurses per 100,000 population was only 102.55 in 2011, although the rate of psychiatric beds per 100,000 population was 204.4, which was, and still is, the highest in the world. ${ }^{19}$ Regulations limit the number of bed in one ward to less than 60 in general hospitals; in psychiatric hospitals, however, wards containing 70 beds are permissible. ${ }^{20}$ During the data collection period, 997 nurses were working in the study wards.

In Finland, the number of nurses working in mental health settings is one of the highest in the world. In 2008, there were 163 nurses per 100,000 population. ${ }^{21}$ Nurses who work in psychiatric hospitals are RNs, specialized nurses, mental health nurses, or assistant nurses. ${ }^{22}$ During the data collection period, a total of 1227 nurses were working in the study wards.

Nurses who worked in psychiatric hospital wards had a certification (RNs, and Assistant Nurses in Japan; RNs, and Mental Health Nurses/Practical Nurses in Finland), and who were willing to participate in the study regardless of age, gender, or years of nursing experience, met the inclusion criteria for our study. Nurses working part-time were also included. Staff members in nursing who worked at out-patient services, child care, forensic psychiatry, or in private healthcare services were excluded from this study. In addition, nurses in managerial positions (Directors of Nursing or Nurse Managers in Japan, and Nurse Managers in Finland) were excluded as well as nurses who were not working during the study period because of sick leave, or maternity leave.

\section{Instruments}

Moral sensitivity. In this study, moral sensitivity was measured using the Revised Moral Sensitivity Questionnaire (RMSQ). ${ }^{23}$ The questionnaire includes nine items answered using a 6-point Likert scale $(1=$ totally disagree to $6=$ totally agree). The factor structure of RMSQ is theoretically assumed to include the following three components: (1) sense of moral burden (four items), (2) moral strength (three items), and (3) moral responsibility (two items). The higher score reflects greater morally sensitive.

In this study, Cronbach's alpha of the RMSQ based on Japanese data were 0.53 for sense of moral burden, 0.72 for moral strength, 0.56 for moral responsibility, and 0.63 for the combined scale. For the Finnish data, they were 0.55 for moral burden, 0.69 for moral strength, and 0.60 for the combined scale. However, Cronbach's alpha of the component "moral responsibility" was very low $(\alpha=-0.03)$. We concluded that the component "moral responsibility" should be excluded, and that the remaining two components of the RMSQ could be used to measure moral sensitivity for this study. As the original RMSQ was written in English, it was translated into Japanese and Finnish. The two versions of the RMSQ were 
back translated and examined, and ascertained to have the same meaning. Translating and using the RMSQ was permitted by the copyright owner, Lützén.

Moral distress. Moral distress was measured by the Moral Distress Scale for Psychiatric nurses (the MDSP). ${ }^{4}$ The MDS-P is based on the original Moral Distress Scale (MDS), ${ }^{5}$ which consists of 30 items related to three factors: (1) individual responsibility, (2) not in the patient's best interest, and (3) deception. While the original scale includes items related to physical care, the MDS-P was adopted to measure moral distress by focusing on items more unique to psychiatric nursing care. Therefore, 23 items from the original MDS were omitted and a 15-item MDS-P was developed with a revised three-factor structure: (1) unethical conduct by caregivers (six items), (2) low staffing (five items), and (3) acquiescence to patients' rights violations (four items). Each item uses a 7-point Likert scale $(0=$ not distressed or no experience to $6=$ distressed intensely). A summary score is formed by summing the scores of the $4-6$ items in each factor with a higher score reflecting a more intense level of moral distress. The MDS-P has been shown to be valid and reliable. ${ }^{4}$ Cronbach's alphas of the MDS-P calculated based on the Japanese data in this study were 0.80 for unethical conduct by caregivers, 0.75 for low staffing, 0.72 for acquiescence to patients' rights violations, and 0.89 for the combined scale; those for the Finnish data were $0.83,0.78,0.74$, and 0.90 , respectively. As these scores revealed that the MDS-P had an acceptable level of reliability, we adopted it to measure moral distress.

The Finnish version of the MDS-P for the present study was translated first from the Japanese into Finnish and then translated into English for comparison to the original English version. ${ }^{7}$ This procedure was repeated as necessary to ensure that the Finnish version of the MDS-P had the same meaning as the Japanese version.

Demographic characteristics. Demographic characteristics collected for the study included nurses' age, gender, qualifications, and years of nursing experience.

\section{Data collection and recruitment}

A hard copy questionnaire was used in both countries to avoid selection bias, ${ }^{24}$ since hard copy is common for questionnaires especially in Japan. In Japan, questionnaires were sent to Nurse Managers in the targeted psychiatric wards. They forwarded the blank questionnaires, information letters, and return-mail envelopes to the Head Nurses, who delivered them to every member of the nursing staff specified during the data collection period. To ensure anonymity, a formal procedure for written informed consent was not used. Instead, the nurses who consented to participate in the study answered the questionnaires voluntarily, put the completed questionnaires into the return-mail envelopes, and then put them into the boxes placed in the wards, which were collected by nurse managers, and then by the researchers. During the data collection period (February-March 2011), 918 completed questionnaires were returned out of 997 questionnaires delivered, giving a $92 \%$ response rate.

In Finland, all nurses working in the 36 study wards were invited to participate in the study. The questionnaires were delivered to contact persons in the hospitals, who forwarded them to the Head Nurses of the wards who then distributed the questionnaires, information letters, and empty envelopes to nurses who fulfilled the inclusion criteria. The response time was 2 weeks (November 2012). Each questionnaire was answered anonymously, which was then sealed in an envelope; it was not possible to identify respondents. The contact persons collected the sealed envelopes and sent them to the research unit. Of 1222 nurses, 988 were given the questionnaires, 974 filled the inclusion criteria, and 534 returned an envelope with a questionnaire. However, 12 questionnaires were either completely blank or included many unanswered items, leaving 522 completed questionnaires for inclusion in the analysis (response rate, 53.6\%). 
Table I. Demographic characteristics of participants in Japan and Finland.

\begin{tabular}{lccc}
\hline & $\begin{array}{c}\text { Japan }(\mathrm{n}=918) \\
\mathrm{n}(\%)\end{array}$ & $\begin{array}{c}\text { Finland }(\mathrm{n}=522) \\
\mathrm{n}(\%)\end{array}$ & $p$ \\
\hline Gender & & & n.s. \\
Male & $275(30.4)$ & $143(27.6)$ & \\
$\quad$ Female & $630(69.7)$ & $375(72.4)$ & \\
Qualification & & & \\
$\quad$ Registered nurse & $637(70.2)$ & $266(52.6)$ & 0.001 \\
Assistant nurse & $264(29.1)$ & $184(36.4)$ & \\
CNS or advanced & $6(0.7)$ & $56(11.1)$ & \\
\hline
\end{tabular}

CNS: certified nurse specialist.

\section{Statistical analyses}

Analyses were performed using SPSS ver. 20.0 and Amos 20. To verify the cultural difference of the moral distress process, a multi-group structural equation model analysis was conducted. This analytical model was a mimic model on the latent variable of moral distress by moral sensitivity with control for some attributive variables (Figure 1). Missing data were handled through full-information maximum likelihood (FIML) estimation. ${ }^{25}$ First, separate models (Model 1), in which no equality constraint was imposed on any parameters across groups, were estimated using the data of each country and confirmed appropriate levels of model fit. Next, we estimated the most constrained model (Model 2), in which equivalency constraint was imposed on all paths apart from the paths of controlled variables. After estimating Models 1 and 2, three structurally invariant models, in which some path coefficients were constrained to be equal across countries, were compared with Models 1 and 2 (see Table 2). This approach allowed us to test whether the structure of moral sensitivity, moral distress, and the relationship between moral sensitivity and moral distress were the same or different between Japan and Finland.

\section{Findings}

\section{Description of the participants}

Demographic characteristics of the Japanese and Finnish nurses are shown in Table 1. In both countries, approximately $70 \%$ of the participants were female. The proportion of qualifications, however, was significantly different; Japan had more RNs and fewer CNSs or nurses with advanced qualifications than Finland. Difference in age varied significantly between Japanese and Finnish nurses $(p<0.001)$. In Japan, nurses' ages ranged from 20 to $82^{\mathrm{i}}$ (mean 44.25, SD 11.75) years, whereas in Finland, nurses' ages ranged from 19 to 64 (mean 41.69, SD 11.33) years. Total of nursing experience and nursing experience in psychiatric settings varied as well: the mean lengths were 18.39 (SD 11.43) years and 12.26 (SD 9.96) years, respectively, in Japan, and 14.43 (SD 10.06) years and 12.96 (SD 10.08) years, respectively, in Finland.

\section{Test of the model}

Table 2 shows the results of multi-group structural equation model analysis. We analyzed the following models: Model 1, with all paths separately estimated between groups; Model 2, with all paths equally

\footnotetext{
${ }^{\mathrm{i}}$ The highest age of the nurses in Japan was unmistakably 82 .
} 
Table 2. Comparison of fit indices among all models.

\begin{tabular}{llccrr}
\hline & & CMIN/DF & AIC & CFI & RMSEA \\
\hline Model 1 & All paths unconstrained & 0.905 & 118.435 & 1.000 & $<0.001$ \\
Model 2 & All paths constrained to be equal & 1.538 & 127.997 & 0.991 & 0.019 \\
Model 3 & Path from MS to MD equal & 0.878 & 116.435 & 1.000 & $<0.001$ \\
Model 4 & Only the paths from MS to the two components equal & 1.070 & 119.542 & 0.999 & 0.007 \\
Model 5 & Only the paths from MD to the three components equal & 1.483 & 128.100 & 0.993 & 0.018
\end{tabular}

MS: moral sensitivity; MD: moral distress. CMIN/DF: Minimum value of C/Degree of Freedom; AIC: Akaike's information criterion; CFI: Comparative fit index; RMSEA: Root mean square error of approximation.

Gender and working environments were controlled as external variable. In addition, their path coefficients were freely estimated in all models.

estimated; Model 3, with only the path from moral sensitivity to moral distress equally estimated; Model 4, with only the paths from moral sensitivity to sense of moral burden, and moral strength equally estimated; and Model 5, with only the paths from moral distress to unethical conduct by caregivers, low staffing, and acquiescence to patients' rights violations equally estimated. The analysis revealed that Model 1 $(\mathrm{CMIN} / \mathrm{DF}=0.922, \mathrm{AIC}=118.435, \mathrm{CFI}=1.000, \mathrm{RMSEA}=0.000)$ provided a better fit than Model 2 $(\mathrm{CMIN} / \mathrm{DF}=1.538, \mathrm{AIC}=127.987, \mathrm{CFI}=0.941, \mathrm{RMSEA}=0.019)$. This implies that our hypothetical model might include cultural differences resulting in different path coefficients between Japan and Finland. When we calculated Models 3-5, only the fit indices for Model $3(\mathrm{CMIN} / \mathrm{DF}=0.878, \mathrm{AIC}=116.435$, $\mathrm{CFI}=1.000$, RMSEA $=0.000$ ) were better than those for Model 1 , while the fit indices for Models 4 and 5 indicated they were not an improvement compared with Model 1 (Table 2). This result suggested that the relationship between moral sensitivity and moral distress was the same between Japan and Finland, but the factor structure of moral sensitivity and moral distress might be different.

\section{Final model}

Figure 2 presents path coefficients for each country (Model 3). As predicted, the results showed that the association of moral sensitivity with moral distress was common and significant in both countries $\left(\mathrm{b}_{\mathrm{j}}=\mathrm{b}_{\mathrm{f}}=\right.$ $0.21, p<0.01)$. All other path coefficients apart from some controlled variables were significant in both countries $(p<0.01)$. On that basis, with regard to moral sensitivity and moral distress, we examined whether the cultural differences of path coefficients were statistically significant using the t-test. The results indicated that the relationship between moral sensitivity and moral strength was significantly different in Japan and Finland $\left(\mathrm{t}=2.26, p<0.05 ; \mathrm{b}_{\mathrm{j}}=1.09, \mathrm{~b}_{\mathrm{f}}=0.56\right)$. Moreover, the relationship between moral distress and low staffing was also significantly different $\left(\mathrm{t}=3.36, p<0.01 ; \mathrm{b}_{\mathrm{j}}=4.83, \mathrm{~b}_{\mathrm{f}}=6.09\right)$. Taken together, these results imply that the association of moral sensitivity with moral distress is significant and similar in both Japan and Finland, whereas the factor structures of moral sensitivity and moral distress are at least partially different between the two countries.

\section{Discussion}

The purpose of this study was to examine the impact of moral sensitivity on moral distress in both Japan and Finland, that is, countries that are geographically distant and have different ethnicities, religions, and cultural backgrounds. The two groups of participants varied in age and qualifications. Ethical beliefs may differ among nurses based on gender and ethnicity, ${ }^{26}$ age and years of experience, ${ }^{27}$ and geographic location. ${ }^{27}$ External factors such as the nurse-to-population ratio also differed between Japan and Finland. 


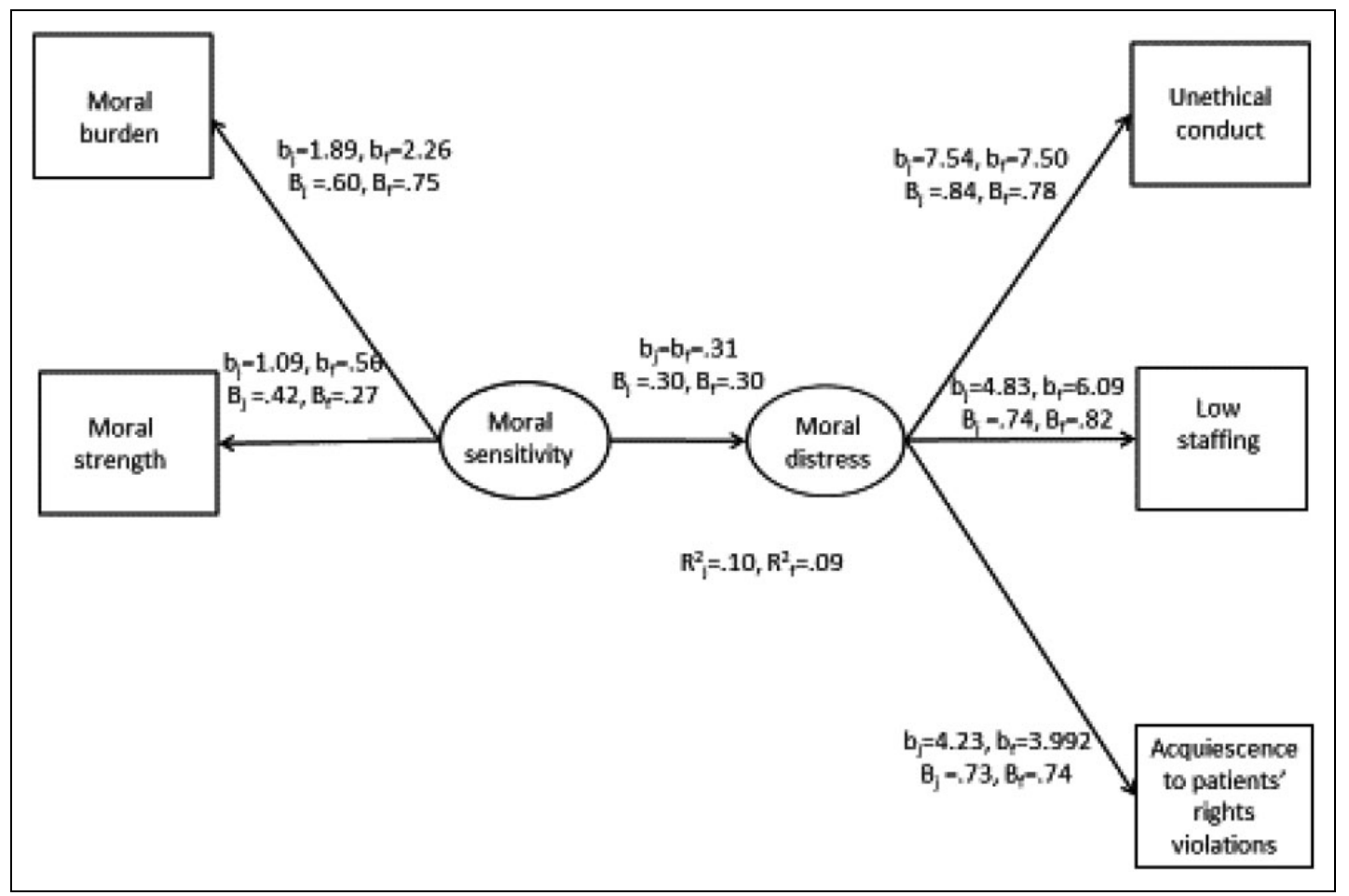

Figure 2. Final structural equation model (Model 3).

" $b_{i}$ " and " $b_{f}$ " are unstandardized coefficients in Japan, and in Finland, respectively, and " $\beta_{i}$ " and " $\beta_{f}$ " are standardized coefficients in Japan, and in Finland, respectively. " ${ }^{2}{ }_{j}$ " is $R^{2}$ in Japan, and " $R_{f}^{2}$ " is $R^{2}$ in Finland. Gender and working environments were controlled for as external variables. Some error terms are omitted. All arrows are significant $(p<0.0 \mathrm{I})$.

Despite these differences, moral distress showed a positive correlation with moral sensitivity among psychiatric nurses, similar to the findings in student nurses ${ }^{11}$ and physicians. ${ }^{10}$ This suggests a general relationship between moral distress and moral sensitivity, in which nurses with higher moral sensitivity experience moral distress more intensely.

Escolar-Chua ${ }^{11}$ found a positive relationship between moral sensitivity and moral distress among student nurses and stated that they "are able to recognize morally distressing and challenging situations which is important is honing their moral sensitivity." The causal correlation, however, was not confirmed because of the cross-sectional design. Student nurses do not yet practice in the same way as nurses, and so do not experience failure to persevere in doing what they believe is right. $\mathrm{Ham}^{28}$ revealed that senior nursing students exhibited higher level principled thinking than experienced nurses and concluded that new graduate nurses abandon personal beliefs of right or wrong to acclimate to the professional work environment. Likewise, Kelly ${ }^{29}$ found that nurses in their first 2 years of practice experienced moral distress as a result of losing personal ideals while trying to adjust to a professional role. It is possible that nurses with high moral sensitivity struggle with morally challenging situations, which leads to moral distress, rather than morally distressing situations honing moral sensitivity.

Since moral sensitivity is required to have a clear understanding of what is really moral, ${ }^{30}$ the results of this study can be interpreted as showing that nurses with a high level of moral sensitivity sense and identify 
moral problems, but cannot cope with them. Lützén and Kvist ${ }^{31}$ stated that "if an individual is not capable of putting his/her moral decision into action, despite his/her moral sensitivity, he/she will experience a more intense moral distress."

The reason why nurses cannot act according to their moral decision (i.e. conquer institutional obstacles) is thought to be their lack of power and authority for changing institutional rules or decision-making. ${ }^{32}$ The Professional Efficacy score among Japanese psychiatric nurses measured with Maslach Burnout Inventory for General Survey was 1.60 , which is rather low. ${ }^{4}$ It is difficult for nurses with a low level of professional efficacy to deal with morally challenging situations.

The value of being morally distressed in itself should not be denied. The American Association of Critical Care Nurses ${ }^{33}$ stated that "Moral distress can lead to a positive change, creating an environment where critical care nurses can make their optimal contribution to the patients and families they serve," which can be applied to other nurses including psychiatric nurses. It is important to prevent nurses with high moral sensitivity from burnout or turnover, and there are several strategies to achieve this. One strategy is to work to change government regulations to increase the nurse-to-patient ratio or improve standards of care. In Finland, the Occupational Safety and Health Act $(738 / 2002)^{34}$ requires that "employers shall continuously monitor the working environment, the state of the working community and the safety of the work practices" (Section 8). In addition, the employer shall systematically and adequately analyze and identify the hazards and risk factors caused by the work, the working premises, other aspects of the working environment, and the working conditions. Typically, supporting employees' well-being at work is a combination of discussions with superiors, model for early support, on-the-job training, and collaboration.

Nurses should, however, take the initiative to improve challenging situations, even if it is difficult for them to change external conditions such as laws and institutional rules. This would become easier if they are advocated for by experts. For this purpose, we have established an online ethics consultation system (http:// www.konan-wu.ac.jp/ rinriconsul/). Professionals such as clinical ethicists, psychiatrists, psychiatric social workers, lawyers, besides mental health nurses are involved as consultants. Any nurse (or anyone) can anonymously consult us about their ethical problems at no charge. The consultees are given replies through emails that contain several strategies they can take, previous examples from other hospitals, persons with whom they should cooperate, rather than a direct or judgmental answer. It is important for nurses with high moral sensitivity to be encouraged to tackle difficult situations without getting burned out. When theses nurses are allowed to pursue what they think is right, it may increase confidence, as well as enhance the level of care and contribute to a more ethical environment.

This study has several limitations. First, study participation was voluntary and participants were not selected randomly, which may bias the data toward those nurses who were willing to share their concerns about moral issues. In Finland, for example, the response rate was low (53.6\%), and therefore, the results may not be representative of all psychiatric nurses in the country. Second, the reliability of the RMSQ scale was low, which might have affected the results of the study as well. The "moral responsibility" component of the RMSQ consists of only two items, which is speculated to contribute to the small Cronbach's alpha. The scale should be examined further using more data. Finally, the data collected in 2011-2012 are rather old, although they are still regarded as valid, since both moral sensitivity and moral distress reflect attitudes that are not likely to change in the short term.

\section{Conclusion}

This study administered a questionnaire survey in Japan and Finland, where ethnicity, culture, religion, or other factors differ to examine the impact of moral sensitivity on moral distress. A multi-group structural equation model analysis was conducted, which affirmed the hypothesis that nurses with higher moral sensitivity will suffer moral distress more than those with lower moral sensitivity. Moral distress in itself 
should not be considered negatively. It is important to remove obstacles, which disrupt ethical practice, by changing the rules and/or the system. It is also important that morally sensitive-hence morally distressed-nurses are encouraged to tackle difficult situations.

\section{Acknowledgements}

We would like to express our appreciation to the nurses participating this study, and the head nurses who helped in the data collection. We also wish to thank the University of Turku, the help provided by Tella Lantta from the Department of Nursing Science, and the late Yumiko Hayama who united the authors.

\section{Conflict of interest}

The author(s) declared no potential conflicts of interest with respect to the research, authorship, and/or publication of this article.

\section{Funding}

The author(s) disclosed receipt of the following financial support for the research, authorship, and/or publication of this article: This study was supported by Japanese governmental fund, JSPS KAKENHI, Grant-in-Aid for Scientific Research (C) 22592585.

\section{References}

1. Jameton A. Nursing practice, the ethical issues. Upper Saddle River, NJ: Prentice Hall, 1984, p. 6.

2. Wilkinson JM. Moral distress in nursing practice: experience and effect. Nurs Forum 1987; 23(1): 16-29.

3. Corley MC, Minick P, Elswick RK, et al. Nurse moral distress and ethical work environment. Nurs Ethics 2005; 12(4): 381-390.

4. Ohnishi K, Ohgushi Y, Nakano M, et al. Moral distress experienced by psychiatric nurses in Japan. Nurs Ethics 2010; 17(6): 721-740.

5. Corley MC, Elswick RK, Gorman M, et al. Development and evaluation of a moral distress scale. J Adv Nurs 2001; 33(2): 250-256.

6. Austin W, Bergum V and Goldberg L. Unable to answer the call of our patients: mental health nurses' experience of moral distress. Nurs Inq 2003; 10(3): 177-183.

7. Ohnishi K, Kitaoka K, Bowers L, et al. Comparison of moral distress and burnout experienced by mental health nurses in Japan and England: a cross-sectional questionnaire survey. J Jpn Health Med Assoc 2011; 20(2): 73-86.

8. Davis S, Schrader V and Belcheir M. Influencers of ethical beliefs and the impact on moral distress and conscientious objection. Nurs Ethics 2012; 19(6): 738-749.

9. Lamiani G, Borghi L and Argentero P. When healthcare professionals can't do the right thing: a systematic review of moral distress and its correlates. $J$ Health Psychol 2015; 22: 51-67.

10. Nejadsarvari N, Abbasi M, Borhani F, et al. Relationship of moral sensitivity and distress among physicians. $J$ Trauma Emer Med 2015; 20(2): e26075.

11. Escolar-Chua RL. Moral sensitivity, moral distress, and moral courage among baccalaureate Filipino nursing students. Nurs Ethics 2018; 25(4): 458-469.

12. Borhani F, Abbaszadeh A, Mohamadi E, et al. Moral sensitivity and moral distress in Iranian critical care nurses. Nurs Ethics 2017; 24(4): 474-482.

13. Jennings B. Mental health service. In: Shannon TA (ed.) Bioethics. 4th ed. Mahwah, NJ: Paulist Press, 2014, p. 2015.

14. Jennings B. Patients' right. In: Shannon TA (ed.) Bioethics. 4th ed. Mahwah, NJ: Paulist Press, 2014, p. 2329.

15. Lo B. Resolving ethical dilemmas: a guide for clinician. 2nd ed. Baltimore, MD: Lippincott Williams \& Wilkins, 2000 , pp. 42-51. 
16. Jennings B. Relativism, cultural and ethical. In: Shannon TA (ed.) Bioethics. 4th ed. Mahwah, NJ: Paulist Press, 2014, p. 2706.

17. Levin KA. Study design III: cross-sectional studies. Evid Based Dent 2006; 7: 24-25.

18. Ministry of Health, Labor and Welfare (MHLW). Byoin hokoku 2015 [reports on hospitals in 2015], 2015 (in Japanese), http://www.e-stat.go.jp/SG1/estat/List.do?lid=000001157930 (accessed 20 April 2017).

19. WHO Mental Health Atlas 2011, http://www.who.int/mental_health/evidence/atlas/profiles/jpn_mh_profile.pdf? ua $=1$ (accessed 1 November 2017).

20. Ministry of Health, Labor and Welfare (MHLW). Kihon shinryoryo shisetu kijun tuchi 2012-02-24 genzaiban [Notice of the standards for basic practice costs, version February 24 2012], 2012 (in Japanese), http:// www.mhlw.go.jp/bunya/iryouhoken/iryouhoken15/d1/5-2-1.pdf (accessed 20 April 2017).

21. WHO health statistics 2014: part III: global health indicators 2006-2010, http://www.who.int/gho/publications/ world_health_statistics/EN_WHS2014_Part3.pdf?ua=1 (accessed 18 August 2013).

22. Ailasmaa R. Kuntien terveys- ja sosiaalipalvelujen henkilöstö 2014. Terveyden ja hyvinvoinnin laitos, tilastoraportti, Personnel in health and social services at municipalities in 2014. National Institute for Health and Welfare, Statistical report, 2015, https://www.julkari.fi/bitstream/handle/10024/126388/Tr16_15_kokonaisraportti.pdf? sequence $=4$ (accessed 2 October 2017).

23. Lützén K, Dahlqvist V, Eriksson S, et al. Developing the concept of moral sensitivity in health care practice. Nurs Ethics 2006; 13(2): 187-196.

24. Tsuboi S, Yoshida H, AE R, et al. Selection bias of internet panel surveys: a comparison with a paper-based survey and national governmental statistics in Japan. Asia Pac J Public Health 2015; 27(2): 2390-2399.

25. Collins LM, Schafer JL and Kam CM. A comparison of inclusive and restrictive strategies in modern missing data procedures. Psychol Methods 2001; 6(4): 330-351.

26. Martin P, Yarbrough S and Alfred D. Professional values held by baccalaureate and associate degree nursing students. J Nurs Scholarsh 2003; 35(3): 291-296.

27. Ulrich CM, Taylor C, Soeken K, et al. Everyday ethics: ethical issues and stress in nursing practice. J Adv Nurs 2010; 66(11): 2510-2519.

28. Ham K. Principled thinking: a comparison of nursing students and experienced nurses. J Contin Educ Nurs 2004; 35(2): 66-73.

29. Kelly B. Preserving moral integrity: a follow-up study with new graduate nurses. $J$ Adv Nurs 1998; 28(5): $1134-1145$.

30. Robichaux C. Developing ethical skills: from sensitivity to action. Crit Care Nurse 2012; 32(2): 65-72.

31. Lützén K and Kvist BE. Moral distress: a comparative analysis of theoretical understandings and inter-related concepts. HEC Forum 2012; 24(1): 13-25.

32. Fry ST and Johnstone M. Ethics in nursing practice: a guide to ethical decision making. Hoboken, NJ: Wiley, 1994.

33. American Association of Critical Care Nurses. The 4A's to rise above moral distress, 2004, http://cmesyllabus. com/wp-content/uploads/2016/03/4As_to_Rise_Above_Moral_Distress.pdf (accessed 18 April 2017).

34. Occupational Safety and Health Act (738/2002), http://www.finlex.fi/en/laki/kaannokset/2002/en20020738 (accessed 29 May 2017). 